\title{
Application of RR Series and Oximetry to a Statistical Classifier for the Detection of Sleep Apnoea/Hypopnoea
}

\author{
AG Ravelo-García ${ }^{1}, J_{L}$ Navarro-Mesa ${ }^{1}$, MJ Murillo-Díaz ${ }^{2}$, JG Juliá-Serdá \\ ${ }^{1}$ Departamento de Señales y Comunicaciones, Universidad de Las Palmas de Gran Canaria, Spain \\ ${ }^{2}$ Unidad del Sueño, Hospital de Gran Canaria Dr. Negrín, Spain
}

\begin{abstract}
In this paper we present a method for the automatic detection of sleep apnoea/Hypopnoea syndrome. This method comprises five steps. These are, signals segmentation, RR series generation, feature extraction, model training and classification. We explore the usage of the RR series and oxygen saturation (oximetry) signals both independently and jointly. Our results show that the joint usage of both improves the results obtained from the use of RR series or oximetry alone. A variety of parameterization techniques are studied in order to extract the relevant features from the signals. For the classification task we propose a two-stage strategy in which epochs are first classified by means of the power ratios. If this classification is not found reliable a Gaussian-mixture-model-based classification is applied in a second stage. A global classification of each subject is given attending to the amount of apnoea epochs. For the experiments we have used 66 subjects. The best results of our method show a 100\% success in the global apnoea classification task.
\end{abstract}

\section{Introduction}

Sleep apnoea is a breathing disorder characterized by brief interruptions of breathing during sleep. Apnoea produces an alteration of the normal sleep arquitecture with multiple arousals which causes somnolence during the day. Sleep apnoea is a serious disease for several reasons: cardiovascular problems, insomnia, somnolence during the day, animic changes and serious social consequences that affects the quality of life of these patients.

The most widely accepted diagnostic test for the sleep apnoea is overnight polysomnography (PSG). The expensive, time-consuming and disturbing nature of PSG prompts many sleep centers to perform an initial screening test in order to reduce the number of PSG [1]. Oximetry $(\mathrm{SaO} 2)$ seems a good alternative to the direct measurement of the upper airway airflow and of the respiratory effort. This is because if one looks for repetitive profound desaturations it is indeed highly specific to apnoea. However, the sensitivity of oximetry with such criterion is generally poor because it is not unusual that patients display normal oximetry during apnoea events (e.g., [2]). Another good alternative comes from the RR series obtained from the ECG signals [3-6]. Such series reflect changes in the airflow. However, these changes are not specific to the apnoeas. In this paper, we study the application of oximetry and RR series to apnoea detection. This is related to the necessity of reducing costs and making easier home diagnosis (e.g.,[2]).

Currently, a diagnosis of sleep apnoea is made by counting the number of apnoea and hypopnoea events over a night sleep in an epoch-by-epoch basis (e.g.,[3]). This is the reason why the first output of our system is a quantification task. That is, each epoch is classified (quantified) as normal or apnoea.

In order to undertake quantification, after preprocessing the RR series, it is necessary to extract its features. A variety of techniques are at disposal for use in RR series and oximetry. We have chosen power-ratiobased coefficient, Autoregresive Coefficients, Filter Bank log-energies and Linear Frequency Cepstral Coefficients because of their ability to extract features from the signals and their potential discrimination capacity.

To carry out the classification task, the classifier we have developed relies on a first stage based on power ratios and a second stage based on Gaussian Mixture Models (GMM) where each class (normal and apnoea) has its own Gaussian mixture. In the test phase, the system provides two outputs. The first one is an epochby-epoch quantification of normal or apnoea. And the second is a global score of the presence of clinically significant apnoea which is derived from the quantification output. A subject is classified as apnoea if the percentage of apnoea epochs is at least $8 \%$.

\section{The Database}

The database was provided by the Sleep Unit of the Hospital de Gran Canaria Dr Negrín. It contains 66 nighttime recordings group (66 subjects with ECG and oxygen saturation signals) of approximately 8 hours duration. For all of them, we have a manual classification of apnoea/ 
Hypopnoea carried out by physicians every 30 seconds epoch. The ECG was sampled at $100 \mathrm{~Hz}$, and oximetry was sampled at $12.5 \mathrm{~Hz}$, each signal having an 8-bit resolution. The subjects of these recordings were men and women between 17 and 72 years of age.

We have defined three classes of recordings with a criterion similar to the one proposed during the Computers in Cardiology Challenge 2000 [7]:

- Group A (Apnoea): Recordings in group A contain at least 200 epochs with apnoea during the recording.

- Group B (Borderline): Recordings in group B contain between 20 and 200 epochs with apnoea during the recording.

- Group C (Control): Recording in group C contain fewer than 20 epochs with apnoea during the recording.

Thirteen recordings are designated as training data (2 group A, 6 group $\mathrm{B}$ and 5 group $\mathrm{C}$ recordings), the remainder of them make up the test data set (17 group A, 16 group $\mathrm{B}$ and 20 group $\mathrm{C}$ recordings). In the experiments Groups A and $\mathrm{C}$ are considered for global classification and all groups for quantification.

\section{Method overview}

We have developed a classification system that comprises the following five steps.

- Signal segmentation: To carry out this work we segment our signals (ECG and oximetry) in 5.5 minutes frames. There are 30 seconds (one epoch) of displacement between adjacent frames for achieving an epoch-by-epoch quantification. This frame length and displacement are a compromise between time and frequency resolution for feature extraction. The central epoch determines the one for which quantification is given.

- RR series generation: Once the ECG signal is segmented, it is band pass filtered between 20 and $35 \mathrm{~Hz}$. Then the signal is full-wave rectified and low pass filtered to $12.5 \mathrm{~Hz}$. Finally, a post processing is applied to eliminate possible errors with a mean filtering of sixth order followed by a median filter of a fifth order.

- Feature extraction: The following features have been tested: Power-ratio-based Coefficient obtained by dividing spectral power in the very low frequency band of the RR series by the total spectral power, Autoregressive Coefficients, Filter Bank log-energies and Linear Frequency Cepstral Coefficients.

- Training: A GMM-based modelling is proposed with a supervised training technique to estimate the models for each class. In the training phase we proceed with the application of the Expectation Maximization (EM) algorithm.

- Classification: In the quantification task we try to determinate the epochs with apnoea. Thus we try to identify the number of apnoea and the percentage that this represents with respect to the total number of sleep epochs. On the other hand, the global classification task discriminates subjects with apnoea and without apnoea. For this challenge, we will make use of the quantification task. Then, a subject will be classified as apnoea if the percentage of apnoea epochs is at least $8 \%$. This percentage means around 5 apnoeas per hour.

\section{4. $\quad$ RR series and apnoea}

Heart rate tends to decrease during the beginning of an apnoea phase and increase once this phase has ended. Every phase coincides with an arousal and an activation of the upper airway muscle permitting the entrance of air in the lungs. Some authors [4] take into account the information that is present in the very low frequency band (VLF, 0.01-0.05 cycles/pulse) of the RR series. The mayor differences between pathological and nonpathological RR series are centered in that band. With this consideration we can understand the importance of a good frequency resolution for our purposes. The segmentation we propose allows a good frequency resolution in the VLF band and a proper feature extraction is possible.

\section{Oximetry and apnoea}

Oxygen saturation signal is also a commonly used polisomnographic recording in the apnoea diagnostic task. If one looks for repetitive (profound) desaturations it is highly specific to apnoea. However, due to the poor sensitivity of oximetry with such criterion we will study the usage of this signal jointly with the RR series. From the spectral point of view, the oximetry has the most part of the energy concentrated between 0 and $0.1 \mathrm{~Hz}$. Thus, it is also important a good frequency resolution.

Figure 1 shows how the apnoea stage is related to oximetry and RR series. This relationship between causes (interruption of the air flow) and effects (changes in oximetry and RR series) allows us to think of extracting the associated characteristics to incorporate them in a classification system.

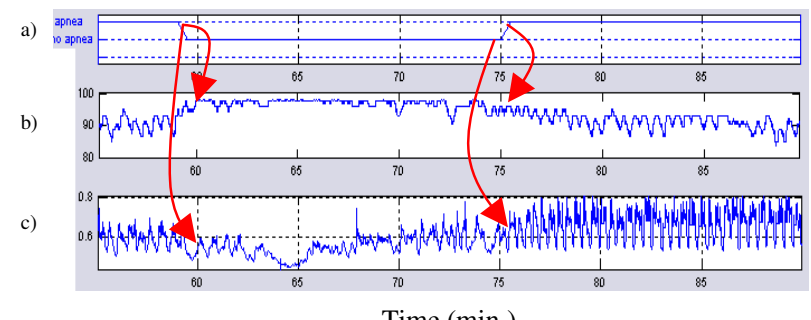

Time (min.)

Figure 1. A subject with apnoea. a) Medical diagnosis. b) Oximetry. c) RR series. 
Figure $1 \mathrm{a}$ ) and b) represent the medical diagnosis and oxygen saturation evolution, respectively, for a recording with apnoea. Between minute 59 and 75 there is no apnoea and oxygen saturation (1b) presents values around 97\%. However, before minute 58 and after minute 76, patient presents desaturations (1b). The drop in oxygen saturation gets to a value around $85 \%$. This drop is acompanied by an encrease in the RR serie

\section{Feature extraction}

A set of features that could potentially be applied for classification were considered. These are:

Power-ratio-based coefficient (PRC). The very low frequency band of the RR series reflects the existence of apnoea events. We use a quotient between the power in the VLF band and the total power.

Filter Banks log-energies (Fbank). We have stated that the presence of apnoea has a clear consequence in the VLF band of the RR series energy. However, we must note that there exist RR series segments where these patterns are not so clearly visible. This situation allows us to suggest an analysis that covers the whole frequency band instead of a portion. A FFT-based bank of equally spaced filters is applied to obtain the energy (or power) at each band. To avoid dependencies with signal dynamics, we normalise each filter output with the total signal power. A logarithm operation is applied to the normalized power values in order to reduce the dynamic range.

Linear Frequency Cepstral Coefficients (LFCC). Discrete Cosine Transform (DCT) has the property of decorrelating power values and reducing the dimensionality of the feature vectors while preserving the relevant information. This fact may be determinant in achieving good classification scores with GMM classifiers. Thus, a DCT-based matrix transformation is applied to the Fbank vectors.

Autoregressive Coefficients (AR). This is a classical method of spectral estimation and modeling. It has been very useful in RR series spectral estimation so it will be used also in our feature extraction process.

\section{Classification system}

The classification system consists of two stages. In a first stage the quantification is made with the PRC features alone. If the recording under test shows a percentage of epochs lower than $4 \%$ then the global classification is normal. However, if this value is exceeded that classification will be omitted, and the recording will be quantified and globally classified using the GMM-based classifier.

\subsection{First stage - power ratios}

In this classification stage the features are extracted from the RR series. A fourteen coefficients AR model showed good performance in estimating a smoothed version of the spectral content in each segment. Then the PRC was calculated. If this coefficient exceeds a determined threshold (e.g., 0.58), the central epoch of the segment is considered as apnoea.

\subsection{Second stage - Gaussian mixtures}

The Power-ratio-based coefficient is a simple and fast measure to obtain an initial quantification. However, it is possible to improve its performance. A Gaussian Mixture Model is proposed as an alternative to PRC. The statistical distribution of the feature vectors for a given class (normal or apnoea) is represented by means of a Gaussian mixture with M Gaussian density functions in the mixture [8]. For the election of the optimal number of Gaussians $\mathrm{M}$, we consider the Bayes Information Criterion looking for a compromise between complexity and performance. With this criterion the number of mixtures per model has been set to 7 in all experiments.

\section{Experiments and results}

In this section we study the application of the feature extraction methods presented in section 6. Except the PRC these methods are applied in the second stage of the classification system.

\subsection{RR-series-based features}

In this section, RR series is analysed individually. The first experiments are intended to study the usage of the PRC in the first stage of the classifier. The AR spectrum is used for estimating the power ratios. The ratio that shows the best result is 0.58 reaching a global classification and quantification rates of $91.89 \%$ and $79.98 \%$, respectively.

In the second experiments we study the second stage of the classifier. Over the whole set of experiments the feature that offers the best result is LFCC with 34 coefficients obtained from Fbank vectors with 36 filters. In addition, we have experimentally found that inserting the PRC as a first element of the feature vector leading to 35 elements improves results. Then, the reached classification rate is $91.89 \%$ for the global classification and $81.18 \%$ for the quantification task (see table 1).

Table 1 Global classification and quantification based on the RR series and oximetry $(\mathrm{SaO} 2)$. Test database

\begin{tabular}{c|cc} 
& Quantification & Global \\
\hline $\mathrm{RR}$ & $81.18 \%$ & $91.89 \%$ \\
$\mathrm{SaO} 2$ & $76.88 \%$ & $91.89 \%$ \\
$\mathrm{SaO} 2+\mathrm{RR}$ & $82.68 \%$ & $100 \%$
\end{tabular}




\subsection{Oximetry-based features}

In this section the oxygen saturation signal will be processed in the second stage of the classifier. The feature that offers the best result is LFCC with 32 coefficients obtained from Fbank vectors with 50 filters operating in the band of interest $(0-0.1 \mathrm{~Hz})$. The reached classification rate is $91.89 \%$ for the global classification and $76.88 \%$ for the quantification task.

\subsection{RR series and oximetry features}

In this case we study the combination of the optimal features of the RR series and oximetry and its integration in the second stage of the classifier. For this classifier a new feature vector will be formed by the union of the best features from the sections 8.1 and 8.2 thus leading to a 67 elements vector. An optimal response is reached with a global classification rate of $100 \%$ and $82.68 \%$ for the quantification task (see table 1). For the training database, the global classification rate is $100 \%$ and quantification rate is $88.22 \%$. It is clear that the combination of RR series and oximetry offers the best performance.

Figure 2 shows a comparison between manual and automatic classification for the test subjects. As we can see, both coincide in global classification. The differences among groups (A,B and C) are clearly visible.

\section{Conclusions}

RR series and oximetry have been individually and jointly studied for detecting apnoea events. With the analysis of these two signals we are suggesting an economic classification alternative intended for achieving the necessity of reducing costs and making easier home diagnosis. A two stage classification system is proposed where a GMM-Based classifier in the second stage has been proven to be a good candidate for improving classification with respect to the power ratios in the first stage. This improvement comes from the fact that Gaussian mixtures are a good way of modelling apnoea events when the features are obtained from the RR series and the oximetry. The combination of oximetry and the RR series improves the results obtained with any of them independently. Feature vectors from LFCC jointly with the PRC show the best results.

\section{References}

[1] [1] Penzel T. Is heart rate variability the simple solution to diagnose sleep apnea?. European Respiratory Journal. June 2003;22;870-871.

[2] [2] Raymond B., Cayton R.M., Chappell M.J. Combined Index of Heart Rate Variability and Oximetry in Screening for the Sleep Apnoea/Hypopnoea Syndrome. J. Sleep Res. (2003)12, 53-61.

[3] Chazal P., Heneghan C., Sheridan E., Reilly R., Nolan P.,
O'Malley M. Automated Processing of the Single-Lead Electrocardiogram for the Detection of Obstructive Sleep Apnoea. IEEE Transactions on Biomedical Engineering, Vol.50, No.6., June 2003.

[4] McNames J.N., Fraser A.M. Obstructive Sleep Apnea Classiffication Based on Spectrogram Patterns in the Electrocardiogram. The Computers in Cardiology Challenge. Computers in Cardiology 2000; 749-752.

[5] Raymond B.Cayton R.M., Bates R.A., Chappell M.J. Screening for Obstructive Sleep Apnea based on the Electrocardiogram. The Computers in Cardiology Challenge. Computers in Cardiology 2000; 27:267-270.

[6] Penzel T., McNames J., de Chazal P., Raymond B., Murray A., Moody G. Systematic comparison of different algorithms for apnoea detection based on electrocardiogram recordings. Medical \& Biological Engineering \& Computing 40:402-407, 2002.

[7] http://www.physionet.org/challenge/2000/

[8] K.D. Bechetti, L. P. Ricotti, Speech Recognition. Theory and C++ Implementation. John Wiley \& Sons, 1999.

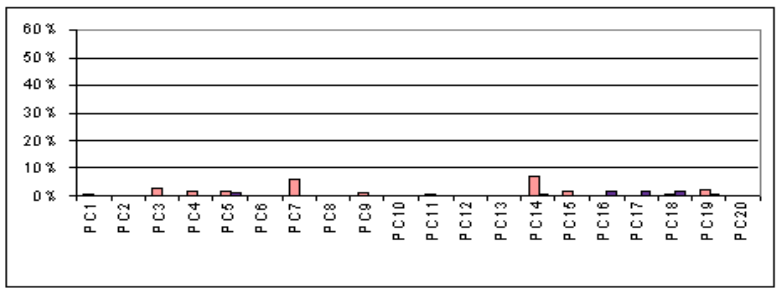

(a)

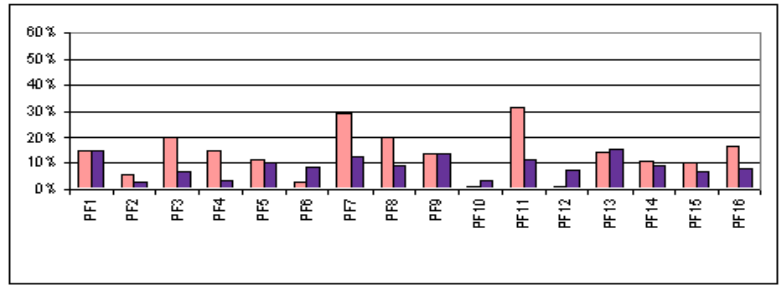

(b)

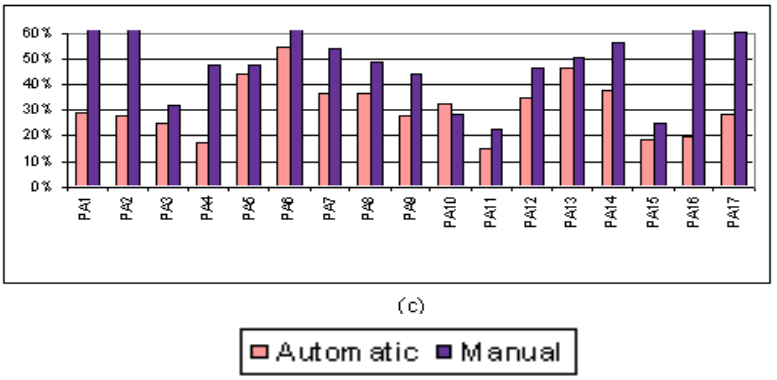

Figure 2. Percentage of epochs with apnoea for subjects of the test database: a) Group $\mathrm{C}$ b) Group B, c) Group A.

Address for correspondence

Juan L. Navarro-Mesa

Pabellón B de Electrónica y Telecomunicación en Tafira. Universidad de Las Palmas de Gran Canaria. 35017 Las Palmas de Gran Canaria. Spain.

E-mail address: jnavarro@dsc.ulpgc.es 